\title{
The Evolution of Cataract Surgery
}

by Geetha Davis, MD

\section{Cataract surgery may be considered one of the most successful treatments in all of medicine.}

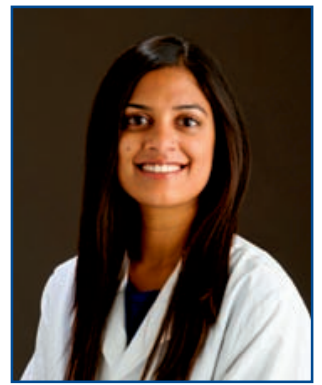

Geetha Davis, MD, MSMA member since 2010 , is assistant professor of ophthalmology and director of the ophthalmology residency program at Mason Eye Institute, Department of Ophthalmology at the University of Missouri.

Contact: raogk@health.missouri.edu

\begin{abstract}
Cataract surgery is one of the most common procedures performed worldwide. It is also one of the oldest. Alongside advancements in cataract surgical techniques have been improvements in intraocular lens replacement technology. Cataract surgery may be considered among the most successful treatments in all of medicine. This article discusses the fascinating evolution of cataract surgery, from the earliest approach of couching to modern day phacoemulsification and lens replacement.
\end{abstract}

\section{Introduction}

The normal crystalline lens of the eye is a clear structure suspended in its natural position by zonular fibers from the ciliary body (see Figure 1). The lens contains a capsule, lens epithelium, cortex, and nucleus. Functions of the lens include refracting light to focus a clear image on the retina and providing accommodation. A cataract is an opacification of the crystalline lens, leading to visual impairment. Many conditions can cause cataract formation. Aging, however, is the most common cause which is multifactorial in nature. ${ }^{1}$ Avoidable risk factors for cataract include use of tobacco products and exposure to ultraviolet radiation. ${ }^{2}$

When a cataract becomes visually significant, cataract surgery is the only established method of treatment. The definition of "visually significant" has evolved over time, to its current meaning of a visual acuity of 20/40 or worse. ${ }^{3}$ When cataract surgery was in its the infancy, a "visually significant" cataract was likely used to describe an advanced or mature cataract, with vision impairment approaching blindness (see Figure 2). Thanks to amazing advancements in surgical techniques and improved safety profiles, the indications and threshold for cataract surgery clearly have shifted to cataract removal at a much earlier stage of development.

\section{Couching and Mature Cataract}

The earliest known method of treating a cataract is couching, which dates back to the fifth century BC (see Figure 3). ${ }^{4}$ The word "couching" comes from the French verb "coucher," which means "to put to bed." Couching was typically performed on mature cataracts. The cataract was not removed from the eye. Instead, the mature cataract was purposefully dislodged out of the visual axis with a needle. The cataract remained in the eye but was no longer blocking light, producing instantaneous improvement in vision. Indeed, in the very immediate postoperative period, couching was considered a success, but the retained cataractous lens and the lack of aseptic technique soon had deleterious effects on the eye, often resulting in blindness shortly after the procedure. Unfortunately, couching is still in practice in some developing countries. $^{5}$ 


\section{Extracapsular Cataract Extraction}

As knowledge of ocular anatomy and eye disease expanded, so did the approach to cataract surgery. While couching is generally thought to have been the predominant method of cataract treatment until the $18^{\text {th }}$ century, ancient literature suggests that as early as $600 \mathrm{BC}$, an Indian surgeon, named Sushruta, may have been the first to perform some type of extracapsular cataract extraction (ECCE). ${ }^{6}$ The procedure is termed "extracapsular" because the lens capsule is left in place. The first true cataract extraction was performed in 1747, in Paris, by the French surgeon Jacques Daviel. His procedure was more effective than couching, with an overall success rate of $50 \%{ }^{7}$ Daviel's procedure basically involved making a large corneal incision (more than $10 \mathrm{~mm}$ ), puncturing the lens capsule, expressing the nucleus, and then extracting the lens cortex by curettage. Although this procedure represented great progress compared to couching, postoperative complications were considerable, including poor wound healing, retained lens remnants, posterior capsular opacification, and infection.

Despite the risks of Daviel's procedure, it remained the accepted approach for cataract extraction for over 100 years, until the $19^{\text {th }}$ century, when intracapsular cataract extraction (ICCE) became, for a time, the preferred method of cataract removal. However, improvements in operative methods and surgical tools eventually led to the reemergence, in the 1970s, of ECCE as the preferred approach over ICCE, which fell out of favor because of high rates of blinding complications. Modern day versions of ECCE and manual small incision cataract surgery (MSICS) are now used in many parts of the world, including the United States. Techniques for performing extracapsular cataract removal have dramatically improved over time, to the point where the overall success rate is now $90 \%$ to $95 \%{ }^{8}$

\section{Intracapsular Cataract Extraction}

In 1753, Samuel Sharp performed the first documented intracapsular cataract extraction (ICCE). ${ }^{9}$ With ICCE, the entire lens, including the lens capsule, is removed through a large limbal incision. Samuel Sharp used his thumb to expel the cataract from the eye. Fracturing the zonular fibers that suspend the lens to the eye was a vital part of the ICCE procedure. The mechanism by which the zonules were broken has evolved from the early use of forceps to hold the lens capsule and manually disrupt the zonules. In 1957 Joaquin Barraquer was the first surgeon to utilize the enzyme alpha-chymotrypsin to dissolve the lens zonules. ${ }^{10}$ Cryoextraction also proved to be a successful method for ICCE. With cryoextraction, a frozen probe is applied to the cataract, which adheres to the probe, and
Figure 1

Sectional view showing the layers of the lens.

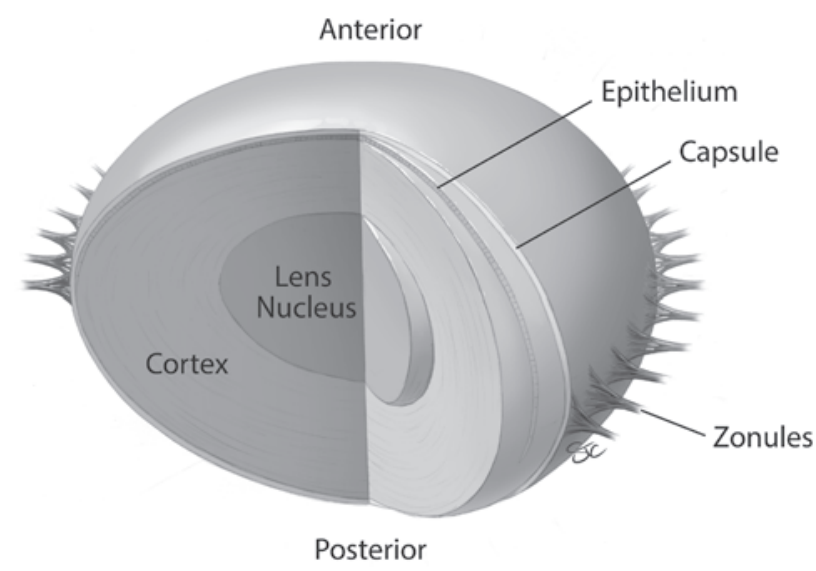

the cataract is gently evacuated from the eye. The success of ICCE grew with the advent of modern anesthetic and sterilization techniques, but its popularity rapidly declined as improvements were made in ECCE techniques. The major drawbacks of ICCE are related to removing the lens and lens capsule in its entirety. The lens capsule serves as a wall between the anterior and posterior structures of the eye. Potentially blinding complications from ICCE, such as retinal detachment, macular edema, and corneal decompensation, are more likely to occur when this wall is not in place to prevent the vitreous from prolapsing forward. Furthermore, ICCE requires larger incisions to remove a cataract, leading to slow healing and a greater amount of surgically induced astigmatism. Despite the drawbacks of ICCE, it remained the primary approach for cataract extraction in the United States, well into the 1970s, and modern ICCE is still in practice in developing countries.

\section{Modern Cataract Extraction and Phacoemulsification}

As improvements in surgical techniques, anesthesia, and equipment evolved, ICCE fell out of favor and was supplanted by ECCE as the standard of care for cataract extraction. ECCE has proved to be an extremely effective method of cataract removal, with considerably better visual outcomes than with ICCE. The advent of an intraocular lens (IOL) to replace the cataractous lens has led to improved refractive results after surgery. The arrival in 1972 of ophthalmic viscosurgical devices (OVDs) improved the facility and safety of the operation as well. An OVD is a gellike substance used during cataract surgery to maintain space in the eye, preventing deflation of the globe and protecting the structures inside the eye without interfering with the steps of the operation. 
In 1967, an American ophthalmologist, Charles Kelman, revolutionized cataract surgery when he introduced phacoemulsification (often referred to as "phaco") as an alternative approach to ECCE. ${ }^{11}$ With conventional ECCE, the entire nucleus of the lens is removed from the eye through a large $(10 \mathrm{~mm})$ incision. In phacoemulsification, an ultrasound-driven needle emulsifies and aspirates the lens through a considerably smaller ( 3 to $4 \mathrm{~mm}$ ) incision. Phacoemulsification was initially met with resistance, but this procedure is now considered the safest and the preferred method of cataract surgery in the developed world. The smaller incision results in a more stable anterior chamber throughout surgery, shorter recovery time, and less surgically induced astigmatism.

The current phacoemulsification procedure is performed in the following manner. Pupillary dilation with topical medications usually takes place in the preoperative holding area. Topical anesthetic agents, introduced by Fischman in 1993, ${ }^{12}$ are commonly administered before the procedure. In the operating room, after sterile preparation of the eye with povidone-iodine and draping, the eye is adequately exposed using a lid speculum, the surgical microscope is positioned, and the surgery begins. A paracentesis, or sideport incision $(1 \mathrm{~mm})$, is made in the cornea. Through this small incision, OVD is injected into the anterior chamber to protect the ocular structures as well as stabilize the globe in preparation for the main incision. The main incision is then made, which can range from $1.8 \mathrm{~mm}$ to $2.75 \mathrm{~mm}$ in size, depending on the instrument used. This wound is constructed in a multiplanar fashion to promote self-sealing at the conclusion of the procedure ("stitchless cataract surgery"). Next, a continuous circular opening is made in the anterior lens capsule (capsulorrhexis) to gain exposure of the contents of the lens. The phacoemulsification handpiece is then introduced into the eye to emulsify and aspirate the lens. After removal of all of the lens contents, all but the anterior portion of the lens capsule is then removed during capsulorrhexis. The anterior portion of the lens capsule, or capsular bag, remains intact and will serve to house the IOL. All of the lens contents are moved by phaco thorugh an opening made in the lens capsule (capsulorrhexis). All that remains after phaco is the lens capsule (minus the anterior portion that was removed during capsulorrhexis). The remaining lens capsule, or capsular gag, will serve to house the IOL.

Phacoemulsification techniques and technology continue to be refined. Precision is paramount. Cataract surgery in developed parts of the world has evolved into a refractive procedure, in which expectations of spectacle independence are commonplace. In the hands of

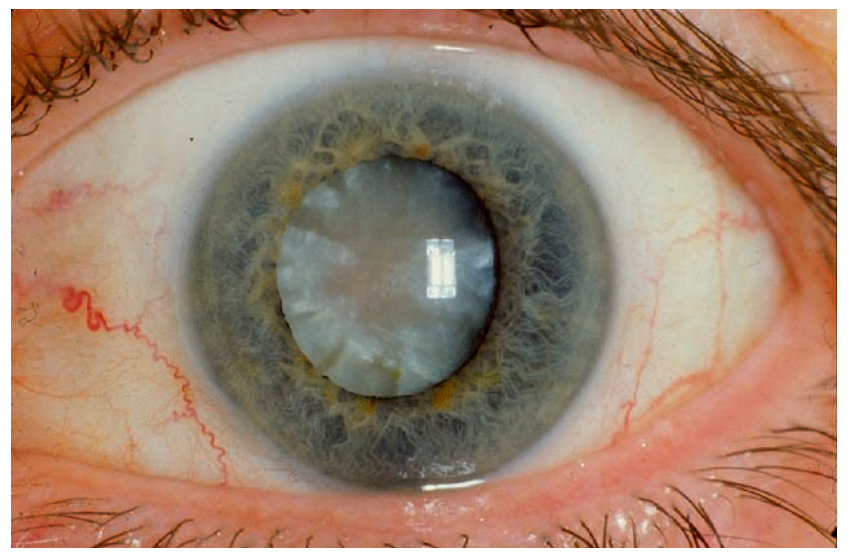

Figure 2

Hypermature age-related corticonuclear cataract with a brunescent (brown) nucleus.

experienced phaco surgeons, the above-outlined steps of cataract surgery are extremely controlled and consistent, so that surgical outcomes are predictable with a good degree of reliability. However, in medicine, there is always room for improvement. In 2001, femtosecond laser technology was applied to LASIK refractive surgery to create the corneal flap, resulting in a more precise LASIK flap. This technology was utilized in cataract surgery for the first time, in 2008, when the first femtosecond laser-assisted cataract surgery was performed in Budapest, Hungary. ${ }^{13}$ The laser does not obviate the need for phacoemulsification. Instead, the laser performs many of the steps of the cataract procedure, including constructing the main wound, creating the capsulorrhexis, and fragmenting the lens. Limbal relaxing incisions to correct astigmatism are often also performed with the laser. With femtosecond laser technology, visual acuity outcomes are thought to be more precise, predictable, and reproducible compared to conventional cataract extraction. However, data do not demonstrate that femtosecond laser-assisted cataract surgery yields outcomes superior to those of manual phacoemulsification surgery. Thus far, the two approaches appear to be equally safe and effective. ${ }^{14}$ Although many cataract surgeons worldwide have adopted femtosecond laser technology, there are still many phaco surgeons who do not believe this technology is beneficial or cost-effective. ${ }^{15}$

\section{Intraocular Lenses}

The incredible success of cataract surgery would not have been possible without the development of intraocular lenses (IOL). In 1949 Sir Harold Ridley, a British ophthalmologist, implanted the first IOL. Prior to the introduction of IOLs, patients were aphakic (without a lens) after cataract surgery. Postoperatively the aphakic 
patient required high-powered hyperopic spectacles to be able to refract light and focus images on the retina. This meant that a patient who was losing vision due to a cloudy lens would have cataract surgery, only to find that, without correction, vision remained poor due to the absence of the lens. A medical student working with Ridley was reported to have asked, Why not replace the cataractous lens with a clear lens? ${ }^{16}$ Sir Harold Ridley realized that wounded World War II pilots tolerated plastic pieces of shattered airplane windshields in their anterior chambers, ${ }^{17}$ and this observation encouraged him to implant an IOL made of polymethyl methacrylate (PMMA), also known as acrylic glass. Initially Ridley's approach garnered little support, as there were considerable postoperative complications, including glaucoma, uveitis, and dislocation of implanted lens.

Remarkable innovations in IOL technology and design have occurred since Ridley's groundbreaking work. The goal of IOL implantation is to achieve the best refractive outcome while avoiding complications. IOLs can be implanted in various sites, including in the anterior chamber, tied to the iris, in the ciliary sulcus (the space between the anterior lens capsule and iris), and in the capsular bag. When IOL implantation was introduced, ICCE was the predominant method of cataract extraction, with removal of the entire lens, including the lens capsule, necessitating placement of the IOL in the anterior chamber or fixed to the iris. Early lens designs were not compatible with long-term safety in the eye. For example, anterior chamber IOLs often spun around in the anterior chamber and damaged the corneal endothelium. Iris-fixated lenses often led to pupillary distortion and uveitis-glaucoma-hyphema (UGH) syndrome.

When ICCE lost its popularity, IOLs were then designed with the intent of posterior chamber implantation. An American ophthalmologist, Steven Shearing, is credited with bringing IOL design into the modern era. In the 1970s he designed a lens that could center itself in the same position where the natural lens was positioned (behind the iris). As advances continued in cataract surgical techniques, a more structurally sound place for the IOL to reside was created: the capsular bag. In 1980 the first foldable lens arrived on the scene, which further improved outcomes. Foldable IOLs are made of flexible material (acrylic or silicone), allowing insertion into the eye through an even smaller incision. Since the 1970s, remarkable advances have occurred in IOL technology, design, and material. In 1992, astigmatism correction became possible when the first toric IOL was developed. ${ }^{18}$ Since then, improvements in the toric IOL model have led to excellent results and increased freedom from spectacle correction.
Figure 3 Couching, the earliest known method of treating cataract.

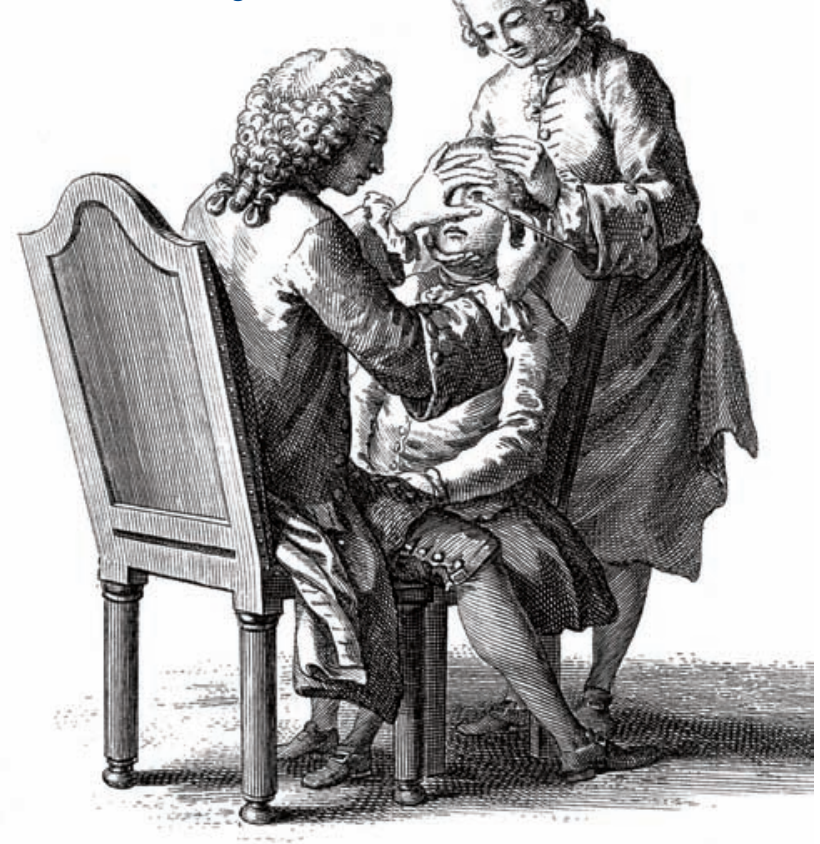

The challenge of correcting presbyopia was taken on with the introduction of multifocal IOLs in the 1990s and of accommodating IOLs in 2000. A multifocal IOL acts like a bifocal inside the eye. It has multiple zones on the surface that will focus distance and near at all times. An accommodating IOL attempts to behave like the natural lens: when viewing an intermediate or near target, the lens is designed to flex forward to focus on the target.

Equally important as lens design is lens power. Just as glasses and contact lens powers need to be measured for each individual, so do IOL powers. When determining IOL power, multiple variables play a role, such as corneal refractive power, anterior chamber depth, length of the eye, and location of the lens when it is placed in the eye. Since IOL implantation became the standard of care in the 1970s, various mathematical formulas taking these variables into account have been utilized to calculate lens power. During the past 20 years, the developments in IOL calculation methods and measuring devices have been astonishing and have resulted in more precise postoperative refractive outcomes. Freedom from spectacles after cataract surgery is now more of an expectation than a mere possibility. Cataract surgeons are constantly evaluating postoperative data to personalize the lens calculation formulas in order to achieve better refractive results. In 2006, a United Kingdom study to establish benchmark standards for refractive outcomes after cataract surgery suggested that over $50 \%$ of patients 
with normal eyes should be within $0.5 \mathrm{D}$ (diopter) of the desired target refraction and $85 \%$ should be within $1.0 \mathrm{D}$ of the desired target refraction. ${ }^{19}$ Studies show that over $90 \%$ of cataract surgery refractive outcomes can be within 1.0D of predicted outcomes and over $70 \%$ can be within $0.5 \mathrm{D}$ of predicted outcome when a surgeon tailors his/her lens calculations using prior outcome data. ${ }^{20,21}$

Even with all of the remarkable technology and mathematical formulas, lens calculations are not perfect. Residual postoperative refractive error can be common. Typically such refractive error is not significant and is tolerated, and can be corrected with glasses or contact lenses if needed. When the refractive error is significant or intolerable to the patient, an IOL exchange may be required. Although an IOL exchange is a safe procedure in the hands of an experienced surgeon, it still subjects the patient to the risks associated with a second intraocular procedure, such as macular edema, vitreous loss, corneal edema, infection, wound leak, and prolonged inflammation. Unlike spectacle and contact lens correction, an IOL cannot easily be "trialed" and exchanged if it is not the correct power. Intraoperative aberrometry is an innovative technology that provides additional data about the eye during the cataract procedure to help in the selection of IOL power and to correct astigmatism. Currently, aberrometry has been found to be most beneficial in achieving the precise alignment of a toric IOL along the axis of astigmatism. ${ }^{22}$

Another exciting development on the horizon is adjustable lens technology, in which the implanted lens power can be adjusted postoperatively with a secondary noninvasive or invasive procedure. There are many adjustable IOL technologies under development, including lightadjustable IOLs, multicomponent IOLs, mechanically adjustable IOLs, and magnetically adjustable IOLs. ${ }^{23}$ This technology will lead to a higher level of precision in cataract surgery.

\section{Conclusion}

Cataract surgery may be considered one of the most successful treatments in all of medicine. With continued advancements in techniques and technology, cataract surgery has evolved into a refractive procedure rather than simply a surgical treatment of cataract. Yet despite the worldwide availability of multiple IOL brands, materials, and models, not all lenses are suitable for every patient (and not all lenses are covered by health insurance companies). While modern cataract surgery has significantly improved the lives of many people throughout the world, perfection seems to be an elusive, moving target. There remains room for improvement, but the future of cataract surgery will be incredible as advances continue to evolve.

\section{Acknowledgment}

Figure 1 by Stacy Turpin Cheavens, MS, CMI, Medical Illustrator, University of Missouri School of Medicine. Figure 2 from National Eye Institute, National Institutes of Health. Figure 3 from Wellcome Library, London.

\section{References}

1. Petrash JM. Aging and age-related diseases of the ocular lens and vitreous body. Invest Ophthalmol Vis Sci. 2013;54:ORSF54-ORSF59.

2. Kelly SP, Thornton J, Edwards R, Saju A, Harrison R. Smoking and cataract: Review of causal association. J Cataract Refract Surg. 2005;31(12):2395-2404.

3. Pascolini D, Mariotti SP. Global estimates of visual impairment: 2010. Br J Ophthalmol. 2012;96 (5):614-618

4. Bobrow JC, Blecher MH, Glasser DB, Mitchell KB, Rosenberg LF, Isbey III EK, Reich J. Surgery for cataract. In: AAO Basic and Clinical Science Source (BCSC) Lens and Cataract (91-161). Singapore: American Academy of Ophthalmology, 2008.

5. Isawumi MA, Kolawole OU, Hassan MB. Couching techniques for cataract treatment in Osogbo, South West Nigeria. Ghana Med J. 2013;7(2):64-69.

6. Grzybowski A, Ascaso FJ. Sushruta in 600 B.C. introduced extraocular expulsion of lens material. Acta Ophthalmologica 2014;92:194-197.

7. Rucker CW. Cataract: a historical perspective. Invest Ophthalmol. 1965;4:377383.

8. Haripriya A, Chang DF, Reena M, Shekhar M. Complication rates of phacoemulsification and manual small-incision cataract surgery at Aravind Eye Hospital. J Cataract Refract Surg. 2012;38(8):1360-1369.

9. Hubbell AA. Samuel Sharp, the first surgeon to make the corneal incision in cataract extraction with a single knife: A biographical and historical sketch. Med Library Hist J. 1904;2(4):242, 1-268.

10. Barraquer J. Drugs and instruments used in cataract surgery. Am J Ophthalmol. 1966;61(1):184-185.

11. Kelman CD. Phaco-emulsification and aspiration: a new technique of cataract removal: a preliminary report. Am J Ophthalmol. 1967;64(1):23-35.

12. Fichman RA. Use of topical anesthesia alone in cataract surgery. J Cataract Refract Surg. 1996;22:612-614.

13. Nagy ZZ, Dunai A, Kránitz K, Takács AI, Sándor GL, Hécz, Knorz MC. Evaluation of femtosecond laser-assisted and manual clear corneal incisions and their effect on surgically induced astigmatism and higher-order aberrations. J Refract Surg. 2014;30(8):522-525.

14. Abell RG, Kerr NM, Vote BJ. Femtosecond-laser assisted cataract surgery compared with conventional cataract surgery. Clin Experiment Ophthalmol. 2013;41(5):455-462

15. Abell RG, Vote BJ. Cost-effectiveness of femtosecond laser-assisted cataract surgery versus phacoemulsification cataract surgery. Ophthalmology 2014;121(1):10-16. 16. Williams HP. Sir Harold Ridley's vision. Br J Ophthalmol. 2001;85(9):1022-1023. 17. Apple DJ, Sims J. Harold Ridley and the invention of the intraocular lens. Surv Ophthalmol. 1996;40(4):279-292.

18. Visser N, Bauer NJ, Nuijts RM. Toric intraocular lenses: historical overview, patient selection, IOL calculation, surgical techniques, clinical outcomes, and complications. J Cataract Refract Surg. 2013;39(4):624-637.

19. Gale RP, Saldana M, Johnston RL, Zuberbuhler B, McKibbin M. Benchmark standards for refractive outcomes after NHS cataract surgery. Eye 2009;23(1):145-152.

20. Garg A, Lin JT, Latkany R, Bovet J, Haigis W. IOL calculation in long and short eyes. In: Mastering the techniques of IOL power calculations. 2nd ed. New Delhi: McGraw-Hill, Jaypee Brothers Medical Publishers (P) Ltd., 2009.

21. Hill, W. IOL Power calculations: How to achieve accurate results. Available at: http://www.doctor-hill.com/iol-main/iol_main.htm. Accessed 5/5/2015.

22. Hatch KM, Woodcock EC, Talamo JH. Intraocular lens power selection and positioning with and without intraoperative aberrometry. J Cataract Refract Surg. 2015;31(4):237-242.

23. Ford J, Werner L, Mamalis N. Adjustable intraocular lens power technology. J Cataract Refract Surg. 2014;40(7):1205-1223.

\section{Disclosure}

None reported. 